Article

\title{
Performance Assessment of Low-Noise Road Surfaces in the Leopoldo Project: Comparison and Validation of Different Measurement Methods
}

\author{
Gaetano Licitra ${ }^{1,2, *}$, Mauro Cerchiai ${ }^{3}$, Luca Teti ${ }^{2,4}$, Elena Ascari ${ }^{4,5}$, Francesco Bianco ${ }^{4}$ and \\ Marco Chetoni $^{2}$ \\ ${ }^{1}$ Department of Lucca, Via A.Vallisneri n.6, Agenzia Regionale per la Protezione Ambientale della \\ Toscana, ARPAT, Lucca I-55100, Italy \\ ${ }^{2}$ Consiglio Nazionale delle Ricerche CNR, IPCF, Via G.Moruzzi n.1, Pisa I-56124, Italy; \\ E-Mails: teti.luca@gmail.com (L.T.); ing.marcochetoni@gmail.com (M.C.) \\ ${ }^{3}$ Agenzia Regionale per la Protezione Ambientale della Toscana, ARPAT, Area Vasta Costa - Settore \\ Agenti Fisici, Via V.Veneto n.27, Pisa I-56127, Italy; E-Mail: mauro.cerchiai@arpat.toscana.it \\ ${ }^{4}$ Physics Department, University of Siena, Via Roma n.56, Siena I-53100, Italy; \\ E-Mails: elena.ascari@unisi.it (E.A.); sephir@gmail.com (F.B.) \\ ${ }^{5}$ Consiglio Nazionale delle Ricerche CNR, IDASC, Via Fosso del Cavaliere n.100, Roma I-00133, Italy \\ * Author to whom correspondence should be addressed; E-Mail: gaetano.licitra@arpat.toscana.it; \\ Tel.: +39-055-530-5493.
}

Academic Editor: Elisabete Fraga de Freitas

Received: 12 November 2014 / Accepted: 4 January 2015 / Published: 15 January 2015

\begin{abstract}
In almost all urban contexts and in many extra-urban conurbations, where road traffic is the main noise pollution source, the use of barriers is not allowed. In these cases, low-noise road surfaces are the most used mitigation action together with traffic flow reduction. Selecting the optimal surface is only the first problem that the public administration has to face. In the second place, it has to consider the issue of assessing the efficacy of the mitigation action. The purpose of the LEOPOLDO project was to improve the knowledge in the design and the characterization of low-noise road surfaces, producing guidelines helpful to the public administrations. Several experimental road surfaces were tested. Moreover, several measurement methods were implemented aiming to select those that are suitable for a correct assessment of the pavement performances laid as mitigation planning. In this paper, the experience gained in the LEOPOLDO project will be described, focusing on both the measurement methods adopted to assess the performance
\end{abstract}


of a low-noise road surface and the criteria by which the experimental results have to be evaluated, presenting a comparison of the obtained results and their monitoring along time.

Keywords: tire/road noise; noise mitigation action; Close Proximity Method (CPX); Statistical Pass-By (SPB)

PACS classifications: $43.50 \mathrm{Rq} ; 43.50 \mathrm{Lj} ; 43.20 \mathrm{Ye}$

\section{Introduction}

Transportation noise is an environmental stressor that causes sleep disturbance and annoyance. The latter is the most frequently ascertained effects of noise for people living in urban areas. The reduction of the urban road traffic noise pollution and of the population noise exposure has become mandatory. A great role in the noise generation mechanism of road infrastructures is played by the road pavement, through the interaction with the rolling tire, which often constitutes the primary source of traffic noise at high speeds [1]. Therefore, the use of road surfaces with low noise emission characteristics is one of the most applied actions all over the world, especially when the source emission must be considered. In fact, the use of noise mitigation solutions based on barriers (e.g., involving only the propagation path) cannot be the only satisfactory solution. There are many cases where a barrier cannot solve the problem at all (e.g., a road in a valley and houses on the surrounding hills) or where it is socially opposed (e.g., in urban contexts). When low-noise road surfaces are used as mitigation actions combined with other actions, as for example traffic planning, it is necessary to have suitable methods to assess the effectiveness of the road surfaces. These methods have to be applicable in all contexts, even where the surrounding conditions are very different from those requested by standards. During last years a great effort was made by some international research projects (such as SILVIA — "Silenda VIA - Sustainable Road Surfaces for Traffic Noise Control"-EU Fifth Framework [2],_-HARMONOISE_-"Harmonized Accurate and Reliable Methods for the EU Directive on the Assessment and Management Of Environmental Noise" [3] — and IMAGINE_- "Improved Methods for the Assessment of the Generic Impact of Noise in the Environment" [4]) to study the traffic road noise sources, developing methods, protocols and models. In Italy, a specific project, called LEOPOLDO, on the evaluation of low noise emission surfaces, started in 2006. In this paper, after a brief description of the LEOPOLDO project objectives, methods and protocols developed are described in detail. Then, results obtained through the time monitoring of the experimental surfaces are reported. Finally, discussions deal with suitability of developed methods, aiming to underline pros and cons of each one, relatively to what should be necessary to evaluate the effectiveness of a road surface laid as mitigation action.

\section{The LEOPOLDO Project}

In Tuscany, the LEOPOLDO project [5] was planned in order to develop innovative noise mitigation techniques to be used in action plans for road infrastructures, based on a new type of pavement layers: the project implementation is part of the required environmental policies to mitigate road noise pollution 
implemented by the Tuscany Region and other European Community member states, in accordance with the Directive 2002/49/EC [6]. The project participants were the Tuscany Region, the ten provinces of the Tuscany Region, ARPAT - the regional environmental protection agency of Tuscany—and the Civil Engineering Department of the University of Pisa. This project was a contact point between public administrators, which have to face noise problems due to road traffic; the environmental agency, which executes noise controls in order to verify the respect of noise limits provided by regulations; and research institutes, which are able to find innovative procedures and suitable mitigation solutions.

\section{Objective}

The aim of project was firstly to study some new kinds of road surfaces. Besides the low-noise aim, the LEOPOLDO project took into account the environmental compatibility of the road surfaces, in terms of raw material, industrial and productive processes, and the safety factors requested by ordinary roads in urban and extra-urban contexts. In addition, economic costs were taken into account, mainly in terms of the proposed solution suitability, which depends on the time-stability of the road surface performances. For example, traffic and weather conditions can cause surface degradation which in turn can cause a decay in noise performance, which leads to the necessity of a new laying. Thus, the LEOPOLDO projects aimed also to find the best surface criteria, based on the surrounding conditions of the whole context in which surfaces were laid, in order to choose the most suitable surface to use (e.g., plain, or hill, or mountain; the presence of ice or snow during winter, or of an amount of water for a long time, and so on).

The secondary aim of the LEOPOLDO project was to develop measurement protocols useful to assess the road surface effectiveness and time stability, in terms of both acoustical and safety characteristics.

Within the LEOPOLDO project, different experimental road surfaces have been laid on six sites, all along regional road infrastructures. Then, they have been characterized using several techniques and monitored during time. Moreover, all sites have been equipped with an instrument able to monitor traffic (amount, speed and type of vehicles), asphalt conditions (temperature, humidity, vertical pressure and strain gauge) and meteorological conditions (air temperature, wind and rain).

A multi-year acoustical monitoring was carried out by means of SPB and CPX methods and results are shown in this paper, and the availability of the experimental road surfaces are a good opportunity to perform further side researches on collected data (for example, see [7-12]), including analysis on vibrations whose elaborations are still ongoing.

Results obtained in the LEOPOLDO project recently lead to the Tuscany Region Guidelines, useful to local administrators for choosing and engineering the most suitable surface to be used as mitigation action on local urban and extra-urban roads [13]. Methods and protocols, developed with the aim to assess the effectiveness of a low-noise road surface laid as mitigation action, have been adopted by the Tuscany Region and their application is requested in all actions based on regional funds [14]. 
Experimental Surfaces

The six experimental road surfaces are detailed in Table 1. All installations are about $200 \mathrm{~m}$ long and placed on extra-urban roads not in densely urbanized areas. For more details on composition, volumetric characteristics, aggregate grading and fractal dimensions of the mixtures see [11].

Table 1. Experimental road surfaces details. For more details see Tables 1 and 2 in [11].

\begin{tabular}{lllccc}
\hline Id & Site & Technology & Bitumen & Depth & Speed Limit \\
\hline 1 & Arezzo & Micro-draining open grade 0/10 & Hard 4.8\% & $3 \mathrm{~cm}$ & $90 \mathrm{~km} / \mathrm{h}$ \\
2 & Firenze & SMA optimized texture gap grade 0/8 & Hard 6.8\% & $3 \mathrm{~cm}$ & $50 \mathrm{~km} / \mathrm{h}$ \\
3 & Lucca & ISO10844 optimized texture dense grade 0/8 & Hard $5.0 \%$ & $3 \mathrm{~cm}$ & $70 \mathrm{~km} / \mathrm{h}$ \\
4 & Pisa & Dense grade 0/6 with expanded clay & Hard $8.5 \%$ & $4 \mathrm{~cm}$ & $70 \mathrm{~km} / \mathrm{h}$ \\
5 & Massa & Micro-draining open grade 0/6 & Hard 4.5\% & $4 \mathrm{~cm}$ & $50 \mathrm{~km} / \mathrm{h}$ \\
6 & Pistoia & Asphalt rubber (wet process) gap grade 0/8 & AR 8.7\% & $3 \mathrm{~cm}$ & $90 \mathrm{~km} / \mathrm{h}$ \\
\hline
\end{tabular}

It is necessary to underline that the experimental surfaces have been laid in contexts which present different local surrounding conditions (lane width, roadside ground, guard-rail, slope, exposure to sun, traffic density and typology etc.). All these differences surely influence the wear and in some cases could invalidate roadside measure comparisons.

\section{Acoustical Analysis: Methods and Developed Protocols}

The acoustical effectiveness of a low-noise road surface is given mainly by the reduction of the tire/road noise, because it is the main noise source of a passing vehicle. Noise reduction can be obtained working on one of the many sound generation phenomena or through absorption across the road surface. Depending on which phenomenon is treated, the noise spectra will turn out different [1]. The main way to reduce the tire/road noise is by lowering the tire vibrations excited by the road texture profile, turning down the whole noise emission spectrum. Another approach is to shift the noise emission peak towards the low frequencies, in order to take advantage from the A-weighting. On the downside, to obtain the shift towards the low frequencies it is necessary a porous surface, and this is not always a well-suitable solution because porosity needs high traffic density and high speed to maintain performances along time. Moreover, the low-frequencies are not taken into account to evaluate noise limits, but they play a significant role in causing annoyance of people [10].

Road surface can also absorb energy along the fist part of the propagation path, providing a further reduction in the roadside levels. Obviously, the aim of a low-noise road surface is to provide lower levels at the roadside (e.g., at buildings façades). Therefore, it is important to evaluate both the tire/road and the roadside noise.

The standard ISO 11819 provides two method to measure the influence of the road surface on the tire/road noise: the Statistical Pass By method (SPB) in the part 1 [15] and the Close Proximity method (CPX) in the part 2 [16]. They have been applied within the LEOPOLDO project and new protocols for measurement and for data post-processing have been developed. In addition, the acoustical impedance have been measured by means of the Impedance tube method, described in the UNI EN ISO 10534 [17,18]. 


\section{1. $S P B$}

\subsubsection{The Method}

The SPB method is described by the ISO 11819-1 and it involves measuring the noise levels from vehicles cruising-by at a constant speed and with the engine operating at the usual condition for that speed. The method relies on a great number of vehicles from normal traffic, without any constraint on tire or vehicle. The measured physical quantity is the maximum A-weighted level $L_{A, \max }$ reached at the microphone positioned $7.5 \mathrm{~m}$ far from the center of road lane, at $1.2 \mathrm{~m}$ height. Data are related to the vehicle speed and the best fit estimates $L_{A, \max }$ value at the reference speed.

Since for every vehicle the levels reach the $L_{A, \max }$ at the microphone when passing over almost the same part of the road, the propagation path is assumed equivalent for all. Therefore, the data dispersion is only due to vehicle models variety, driving behaviors and mainly tire variety [Phillips-Abbot]. To further improve the data accuracy, vehicles are classified in some categories depending on the weight and the number of tires/axles. Then, the SPB indexes are calculated as a linear combination of the $L_{A, \max }$ values at the reference speed obtained for different categories. The standard prescribes that microphone has to be positioned without reflecting obstacles neither behind nor laterally. Moreover, vehicles should pass at constant speed and between microphone position and the center of the road lane there should be the same surface. These surrounding conditions often avoid the applicability of the SPB method in urban contexts.

\subsubsection{The Modified Protocol}

The protocol applied by ARPAT within the LEOPOLDO project combines the technical international standard with the guidelines provided by HARMONOISE project [19]. HARMONOISE introduces a second measurement position, at $3.0 \mathrm{~m}$ height, at $7.5 \mathrm{~m}$ far from the center of road lane, to improve the evaluation of the influence of local context, avoiding the roadside ground influence not negligible in case of the $1.2 \mathrm{~m}$ height position. Moreover, the applied procedure is based on measuring the acoustical energy of the passing vehicle, using the sound exposure level (SEL) in place of the $L_{A, \max }$ prescribed by the ISO. The SEL is calculated in according to the ISO 1996-2 [20], which defines the pass-by event as the signal part in which level exceeds the background noise more than $10 \mathrm{~dB}(\mathrm{~A})$. Thus, during the measurement session, pass-by sound pressure signal and related speed are registered.

In the post processing analysis, the statistical sample of many single passages constitutes the data-set for a logarithmic regression (1), between the measured speed $v$ and the SEL for each microphone to estimate the level at the reference speed $v_{0}$, in accordance with the HARMONOISE project.

$$
\mathrm{SEL}=A+B \log \left(\frac{v}{v_{0}}\right)
$$

where $A$ is the SEL at reference speed $v_{0}$ (tipically $50 \mathrm{~km} / \mathrm{h}$ ) and $B$ is a speed-related correction.

Due to the traffic densities and typologies that characterize experimental sites, only the light vehicles category is significantly populated. Thus, only the SPB index for light vehicles, named $L_{1}$ level, is calculated. 
However, the SPB procedure fails when speed data gather around a specific value (commonly the speed limit): in fact, when the speeds are almost the same, the variability due to driver behavior and to vehicle characteristics dominates and data constitute a cloud. In this case, data outside the cloud influence the fit algorithm. To avoid the influence of possible outliers outside the cloud, the binning technique was applied to the whole data-set and a minimum chi-square fit of central values with their uncertainties is performed. That is: data are grouped in velocity classes - called bins-about $10 \mathrm{~km} / \mathrm{h}$ wide (the actual width is chosen in order to minimize the total chi square of the final fit); mean and standard deviations of data $L_{\mathrm{EQ}}(\mathrm{A})$ in each class are computed with the hypothesis of a Poissonian distribution of data and each class is represented by the tern: center velocity of the class; mean $L_{\mathrm{EQ}}(\mathrm{A})$ and standard deviation of data contained in each class. The terns are used in the best fit between $L_{\mathrm{EQ}}(\mathrm{A})$ and velocity. In physical statistics this technique is usually called binning.

In this way, the information of data spread out and the numerousness in each speed bin is taken into account by means of the uncertainty associated with the central values. In Figure 1 an example of data binning is provided.

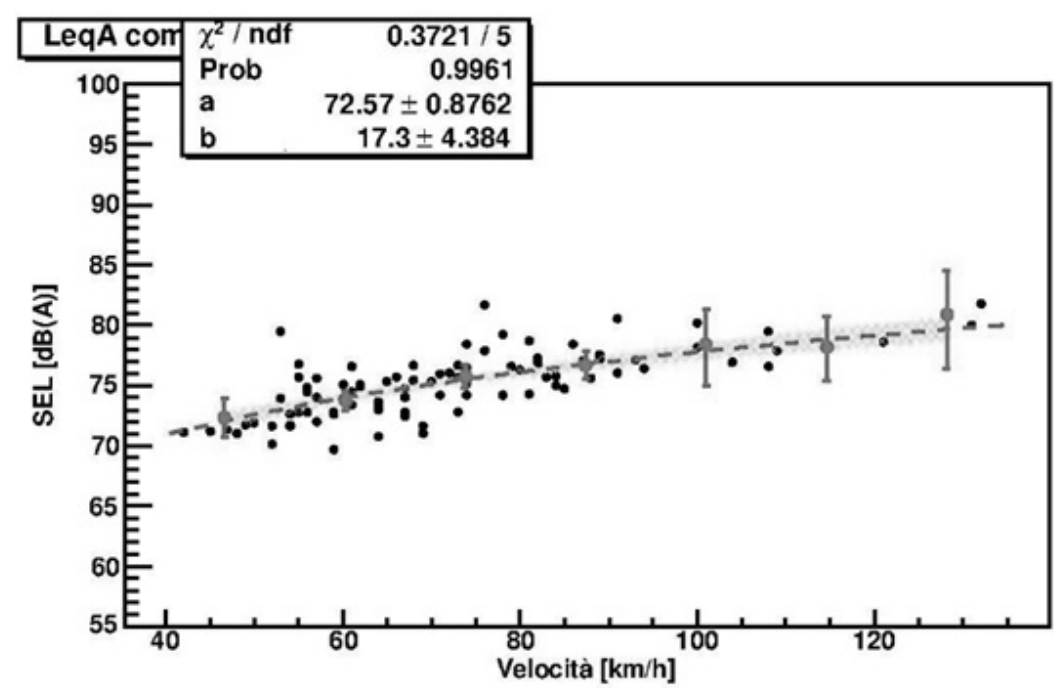

Figure 1. Example of fit performed with binning technique.

\section{2. $C P X$}

\subsubsection{The Method}

The CPX method is described in the standard draft ISO/DIS 11819-2 (2011) [16]. The CPX measurements can be carried out using a trailer towed by a separated vehicle or a self-powered vehicle. The method uses two microphones placed at $0.2 \mathrm{~m}$ from the axis of the wheel and $0.1 \mathrm{~m}$ above the road surface. The microphones position is chosen in proximity to the tire/road contact, aiming to evaluate only the road-tire noise without the engine and exhaust system of the car.

The use of four reference tires was requested by the 2002 ISO 11819-2 release, but in the actual one number and technical specifications are committed to the third part actually unwritten. For standard application some runs (at least three) on both wheel tracks are requested. For particular applications, only one single run, carried out on the wheel track closest to the edge of the road and using only one reference tire is allowed. The measurement protocol requires that the sound signal over $20 \mathrm{~m}$ 
long road segments together with the corresponding vehicle speeds are recorded. Then, for each road segment, the A-weighted equivalent third-octave-band level from $315 \mathrm{~Hz}$ to $5000 \mathrm{~Hz}$ is determined at each microphone position. The energy-based average sound level for the two microphones, normalized at the reference speed by a simple correction procedure or by a logarithmic regression between levels and speed data, is called "Tire/road Sound Level" $\left(L_{C P X}\right)$. Finally, the road surface, preferably longer than $100 \mathrm{~m}$, is characterized averaging all the $20 \mathrm{~m}$ long segments, while the standard deviation around the mean is an indication of the homogeneity.

\subsubsection{The Modified Protocol}

An adapted protocol for measurement and data post-processing has been developed to improve the suitability of the CPX method within the LEOPOLDO project [21]. In the present work, results are shown in terms of tire/road noise levels, without strictly referring to CPX indexes; however, for the sake of simplicity, they are hereafter referred as $L_{C P X}$ values. The set-up is based on the measurement system mounted on a self-powered vehicle, as described in [22-24], using the Green Michelin Energy XH1 185/65/R15 as reference tire. In the post-processing step, data analysis is based on the spatial resolution of $5.84 \mathrm{~m}$ long segments and the sound pressure level $L_{p_{i}}$ associated to the $i$-th segment is estimated by fitting experimental data by the well-known bi-logarithmic relationship with speed data. The fit is calculated for each segment, for each third octave band level in the frequency range of 315 to $5000 \mathrm{~Hz}$. It is computed using a minimum chi-squared based iterative algorithm, taking into account the asymmetry of the uncertainties derived from the logarithmic conversion. Finally, the overall A-weighted equivalent sound pressure level, at the reference speed, associated to the $i$-th segment, $L_{C P X_{i}}$, is obtained through the A-weighted energy-based sum of the third octave bands estimated levels, as required by the ISO.

The $L_{C P X_{i}}$, levels versus distance are used to characterize the road surface installation through its homogeneity and the averaged noise levels on all segments.

The last improvement of the modified protocol prescribes that during the same measurement session runs have to be extended over a second road surface, typically a DAC 0/12 or a SMA 0/12, as suggested in [21], close to the test one as much as possible. The selected second surface then becomes the "reference" and the evaluation of the acoustical performances of the test one is carried comparing it to the reference one. This reference surface could be as equal as possible to the pre-existing, ante-operam one (e.g., long aged and possibly acoustically stable in time), or, alternatively, a road surface coeval to the test one. This choice depends on the purpose of the measurement or the aim of the test surface laying. This procedure, called "the differential criterion", came from the necessity to avoid influence by measurement conditions (especially meteo-climatic ones). Their effect would depend on the particular configuration tire/road and in real scenarios it is nearly impossible to find the appropriate correction for each surface surveyed. Moreover, despite in a single measurement session most of these error sources affect systematically the measurements, they can be assumed as random in case of several measurement sessions carried out in different days and/or with different set-ups or instrumental chains. Thus, comparing absolute values obtained could be not significant. 


\section{Experimental Results}

In each site, the SPB method was applied firstly on the pre-existing surface (ante-operam one), before the laying of the experimental surface, carrying out the measurement session according to the developed protocol above explained. Thus, results obtained later for the experimental surface were compared to the ante-operam one. This allows to evaluate over time the effectiveness of the experimental road surface relative to the pre-existing status (e.g., considering the new laying as a mitigation action).

In case of the CPX method, the differential criterion above described, allows to avoid the use of results obtained in measurement sessions carried out before the experimental laying.

Concerning the uncertainties evaluation, it has to be considered that: for the SPB results, data variability derives exclusively from the road traffic, because the road spatial unhomogeneity influences in the same way each pass-by. Thus, the uncertainty associated to results, calculated through the SPB fit algorithm, depends on the sample and it can be considered as a measurement uncertainty; on the contrary, the uncertainty associated to the CPX results derives mainly from data dispersion (e.g., the spatial unhomogeneity of the road surfaces). Thus, CPX results obtained in different measurement sessions carried out on the same road surface, shall show almost equivalent uncertainty, unless unhomogeneity was increasing. These observations are important to better evaluate results reported below.

\subsection{Site 1-The Micro-Draining Open Grade 0/10}

The road is characterized by high traffic density, with a significant percentage of heavy vehicles and an average speed of about $80 \mathrm{~km} / \mathrm{h}$. Lanes are wide with good visibility on the test stretch, which is surrounded by containment walls. The experimental road surface is porous and it shows an absorbing peak around $1100 \mathrm{~Hz}$, as shown in Figure 2.

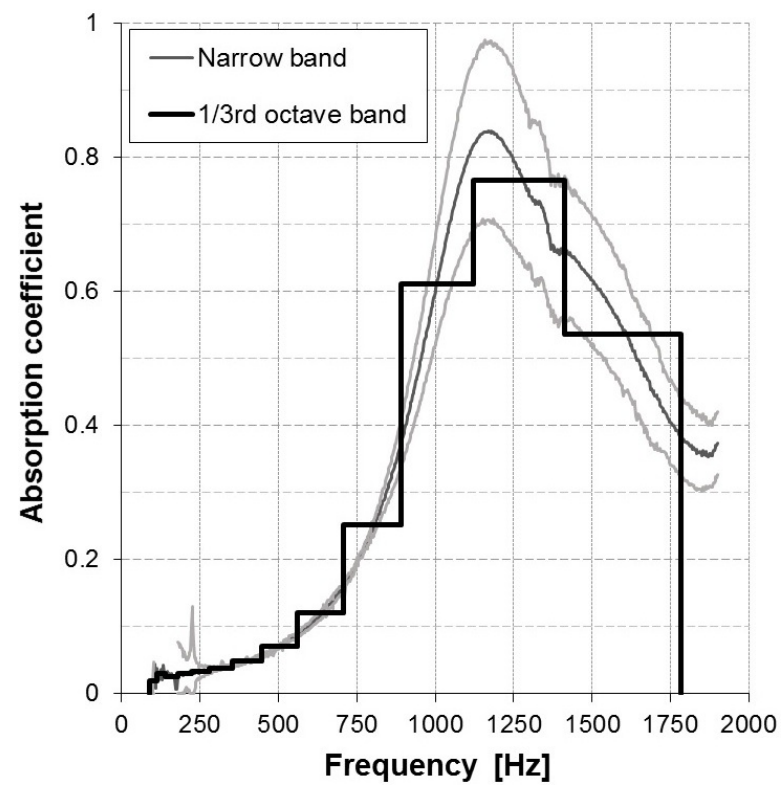

Figure 2. Site 1: Absorbing coefficient vs. frequency measured using impedance tube on some samples extracted from the surface. In grey the dispersion of data from narrow band analysis and in black the resulting 1/3rd octave band result. The main absorption peak can be found at about $1100 \mathrm{~Hz}$. 
The CPX results along time are shown in Figure 3. In Figure 3 a absolute $L_{\mathrm{CPX}}$ values for reference and experimental surfaces are plotted, while in Figure $3 \mathrm{~b}$ their difference is plotted for each lane.

In this site, the reference surface is coeval with the experimental one. Looking at the absolute values, the reference surface can be probably considered acoustically settled after the second year. On the experimental road surface, the acoustical characteristics of a lane 1, (continuous line in Figure 3b) are getting worse before the other one lane 2, (dashed line in figure).

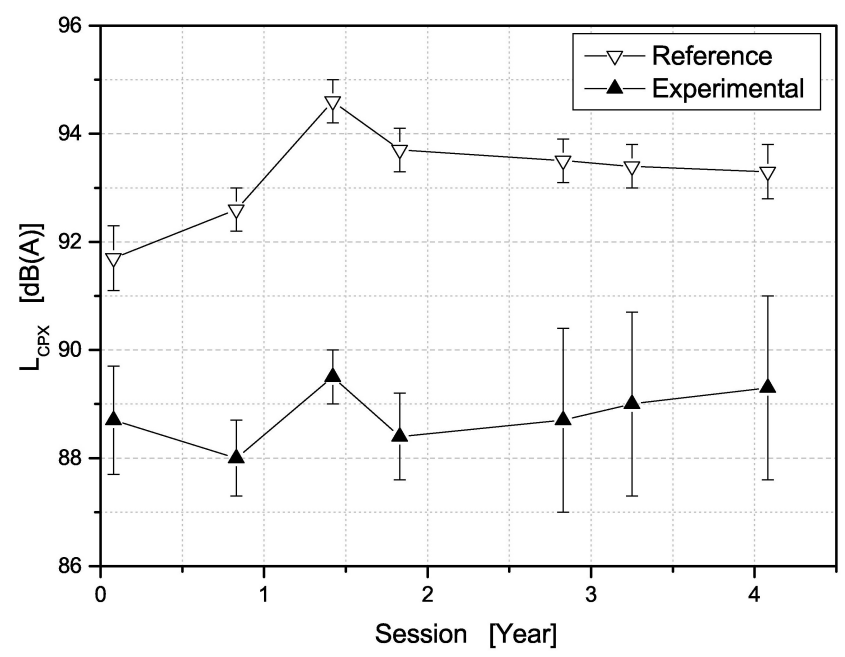

(a)

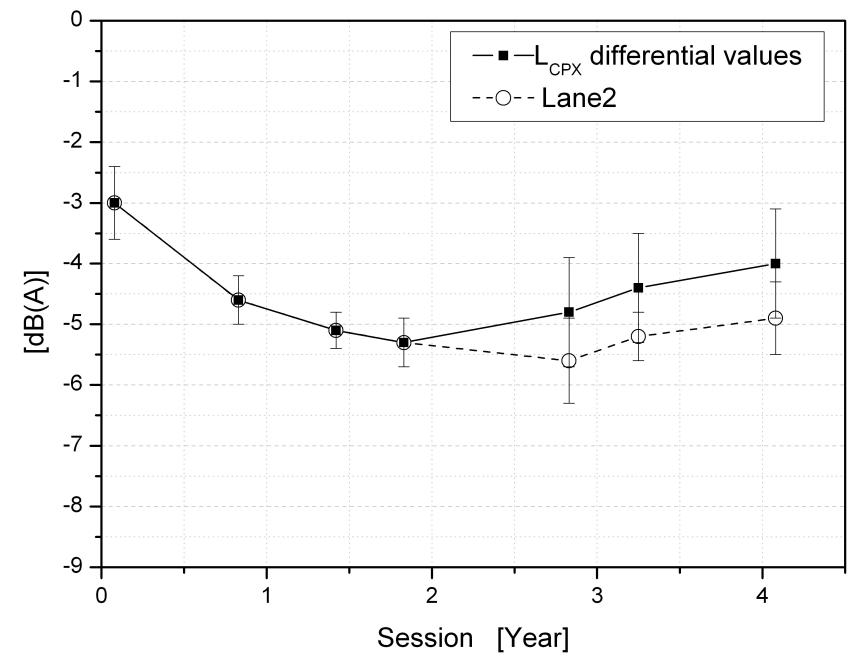

(b)

Figure 3. Absolute (a) and differential (b) values of $L_{C P X}$ along measurement sessions (e.g., time, in years) for the micro-draining surface laid in Site 1.

This can be figured out in Figure 4, where the spatial distributions of $L_{\mathrm{CPX}}$ levels of both lanes are plotted, pointing out differences between the first and the last measurement session carried out on the experimental surface. Since the first session lane 1 shows a higher spatial unhomogeneity than the lane 2. The hypothesis that the laying of the lane 1 suffered some troubles (in the temperature, in the mix or other) is clearly confirmed by results obtained in the last measurement session, where it is worsened in terms of both spatial homogeneity and absolute values. This is also highlighted in the $L_{C P X}$ absolute values graph, where the uncertainty for the experimental road surface is increasing in the last measurement sessions, and in the differential values graph, where also results obtained considering only the lane 2 are plotted.

The SPB measurements were carried out on the lane 1 and in its first part(referring to the Figure 4, the stretch centered on the $150 \mathrm{~m}$ in the plot), so the increasing roadside level is justified. Moreover, comparing absolute values it can be easily noticed that the third measurement session (see Figure 5), about one year and an half after the laying, has been affected by some bias evidently due to surrounding condition (given that CPX and SPB measurement are carried out through two different instrumental chains and by two different couples of operators), probably the not perfectly dry road surface because the day before the measurement session it was a rainy day. It is remarkable as the differential criterion is powerful to minimize the influence of this bias on results. 


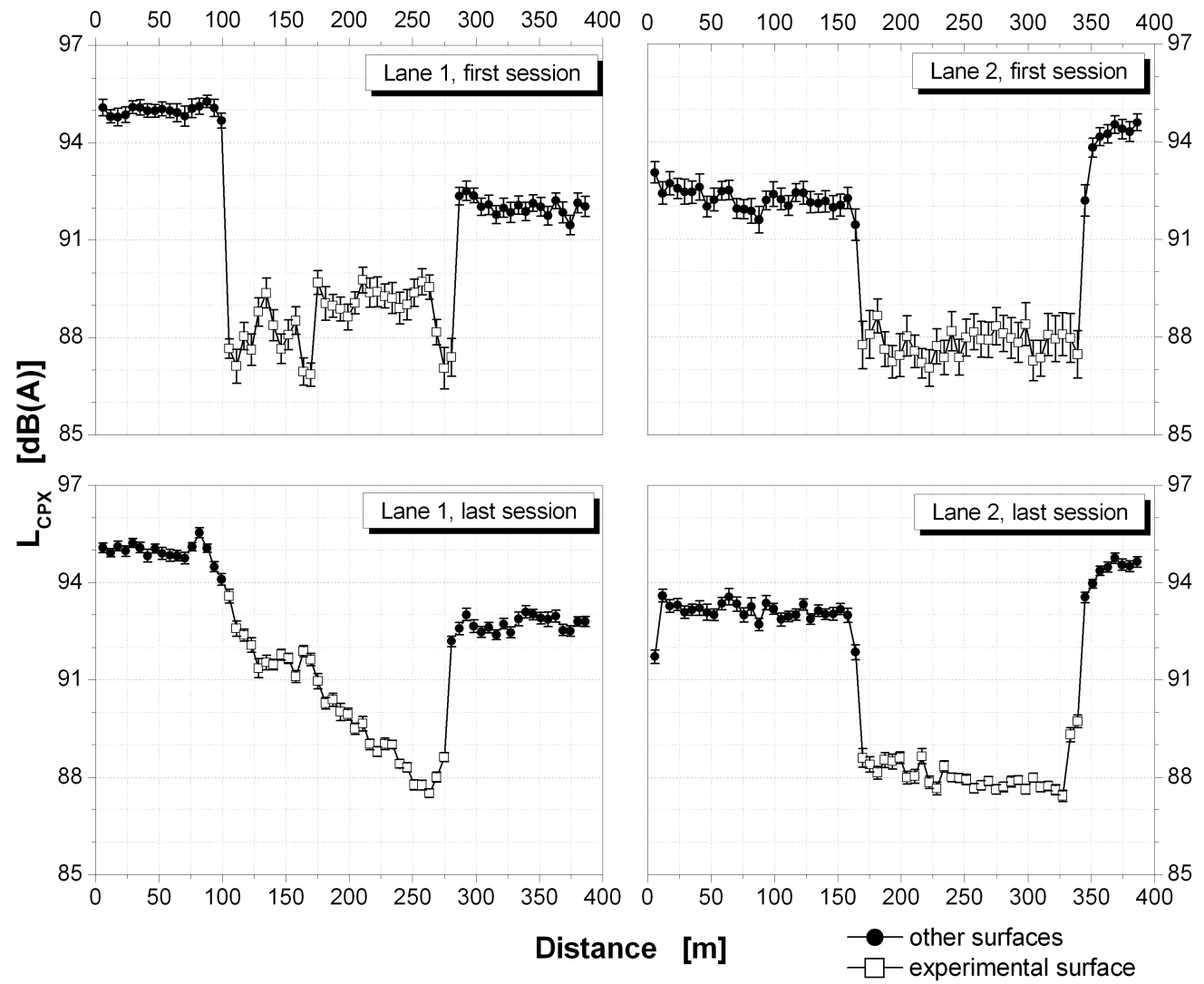

Figure 4. CPX data spatial distribution obtained during first session (upper figures) of measurements and during last one (lower figures) at Site 1. Lane 1 results are represented on the left plots while lane 2 ones are on the right plots. Experimental stretches data are marked with white squares.

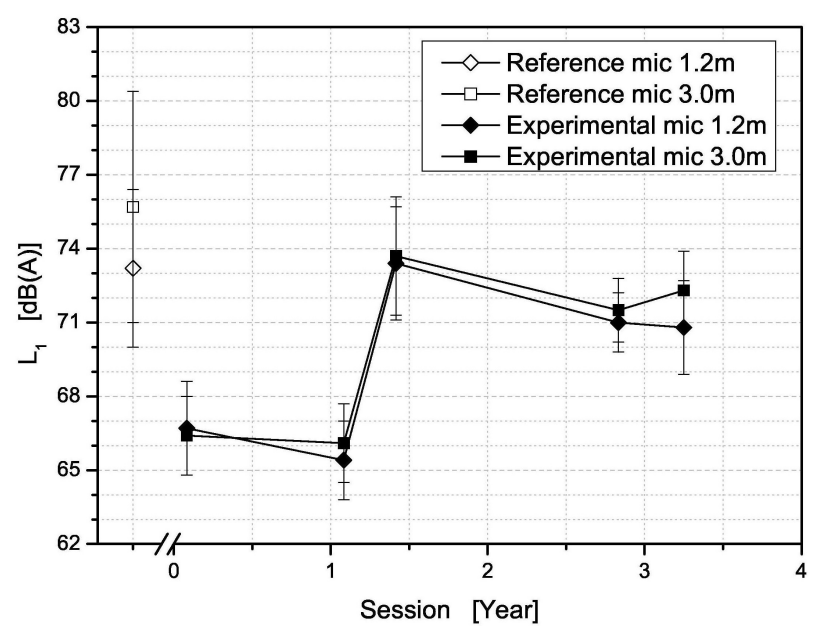

Figure 5. Time evolution of SPB values from lane1, Site 1. In white ante-operam values.

\subsection{Site 2-The SMA Optimized Texture Gap Grade 0/8}

The road is characterized by low traffic density, with limited percentage of heavy vehicles and with an average speed of about $70 \mathrm{~km} / \mathrm{h}$. There is no good visibility along the site, going from a bend to a 
nearby small slope. A hill is present on one side of the road, while the other one has a descending slope with some buildings.

The CPX results are shown in Figure 6, the SPB results are shown in Figure 7. In this case, results show that, after the first year characterized by an initial settling, the acoustical characteristics of the road surface are almost stable around a $3 \mathrm{~dB}(\mathrm{~A})$ lowering of tire/road noise emission. It is clearly highlighted by the $L_{C P X}$ differential values and confirmed by the SPB ones. Moreover, it can be easily noticed that the 2 year aged measurement session was affected by some bias due to surrounding condition, confirming that the differential criterion is powerful to minimize external influence on results.

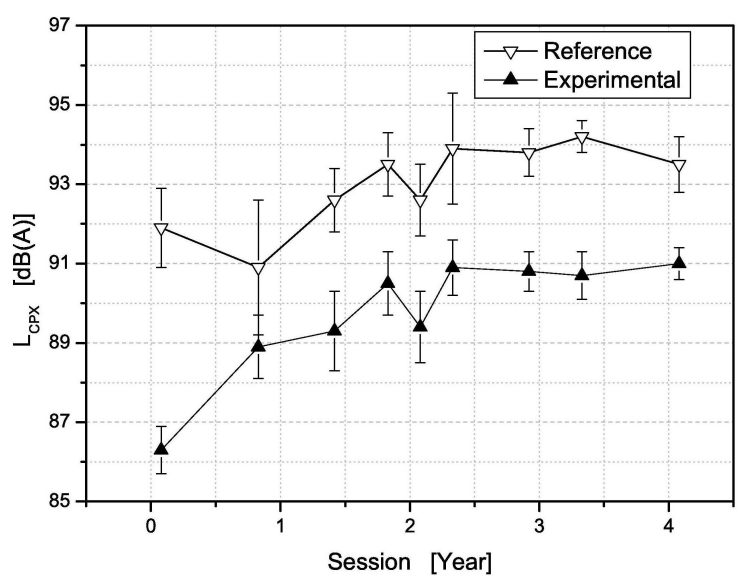

(a)

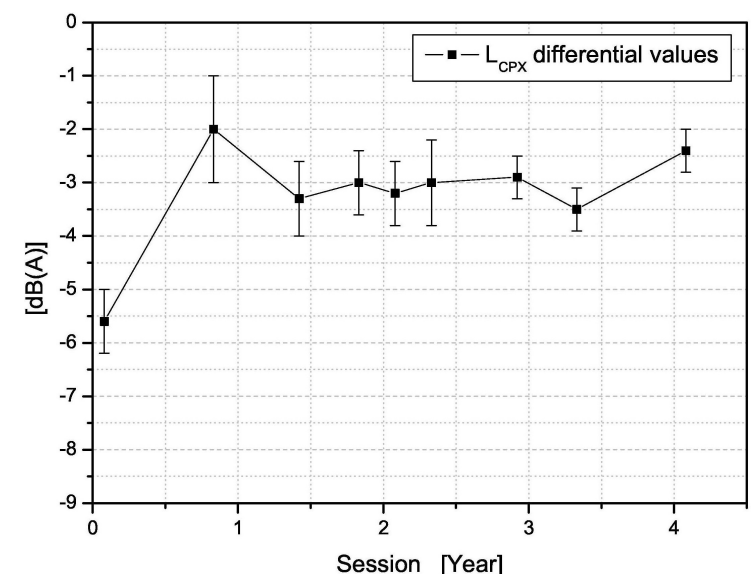

(b)

Figure 6. Absolute (a) and differential (b) values of $L_{C P X}$ along measurement sessions (e.g., time, in years) for the SMA optimized texture surface laid in Site 2.

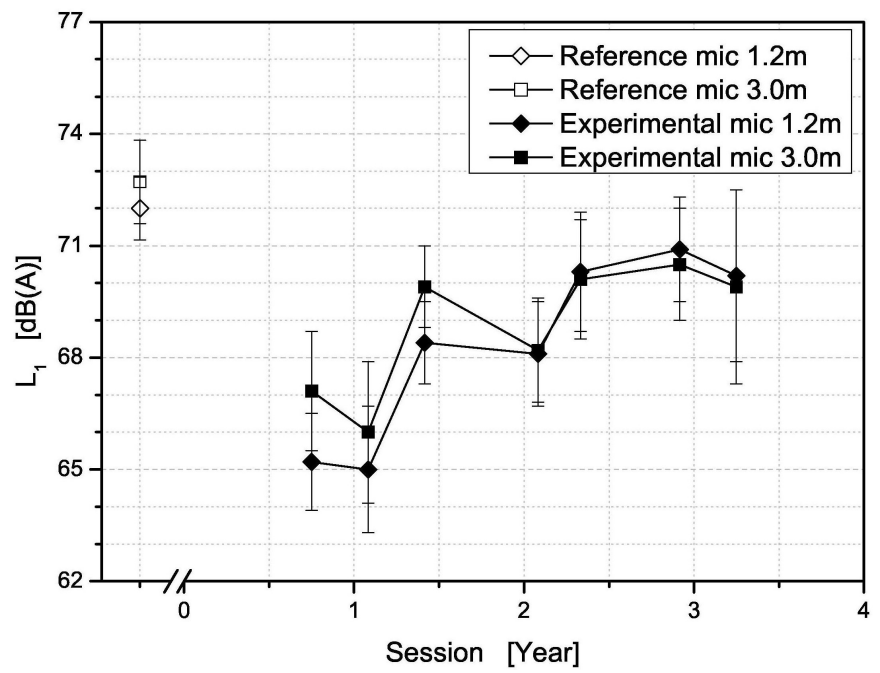

Figure 7. Time evolution of SPB values in Site 2. In white ante-operam values.

In Figure 8 the CPX data spatial distribution of both lanes is shown, pointing out differences between the first and the last session on the experimental surface. Probably, the lane 2 in the first session was still settling and loosing the outward bitumen in excess, showing an unhomogeneity unascertainable in 
the last measurement session. There are not large differences between the two lanes, and both show an equal increasing of levels.

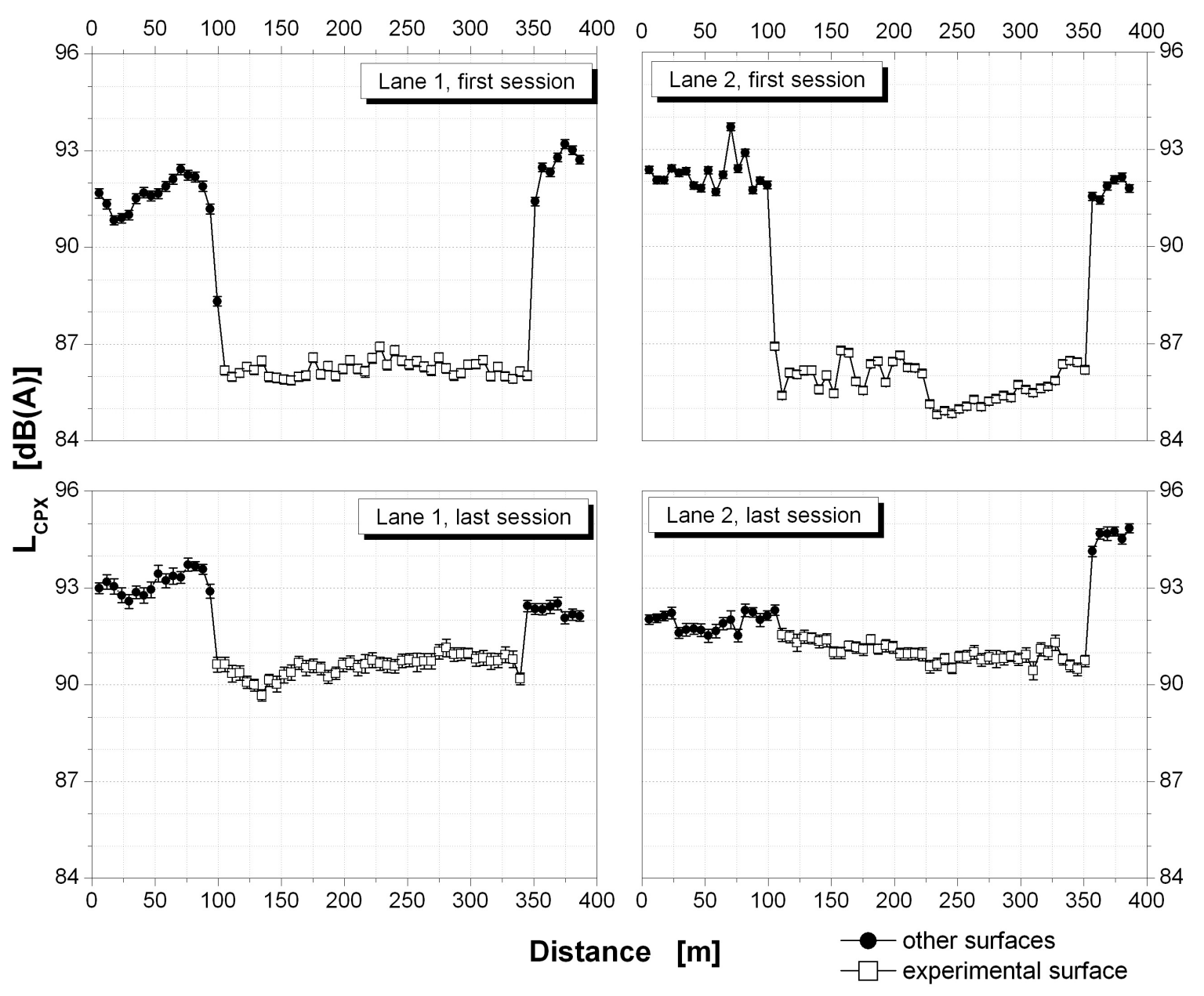

Figure 8. CPX data spatial distribution obtained during first session (upper figures) of measurements and during last one (lower figures) at Site 2. Lane 1 results are represented on the left plots while lane 2 ones are on the right plots. Experimental stretches data are marked with white squares.

\subsection{Site 3-The ISO10844 Optimized Texture Dense Grade 0/8}

This site shows a not high traffic density, with limited percentage of heavy vehicles and an average speed of about $50 \mathrm{~km} / \mathrm{h}$. There is a good visibility along the site and the road has a small slope. On one side of the road there is a steep slope side of a hill, whilst on the other side there is a stream $5 \mathrm{~m}$ further down after the small flat lawn where the SPB instrumentation is placed. The CPX results are shown in Figure 9. In this case, results show that in the first 18 months the road surface has been characterized by an initial settling. On the other side, since the second year from the laying, the acoustical characteristics of the road surface are almost stable around a $5 \mathrm{~dB}(\mathrm{~A})$ lowering of tire/road noise emission.

The CPX spatial distribution, shown in Figure 10, points out its homogeneity and its stability in time: in fact, since the first measurement session and till the last one lanes show equivalent levels. 


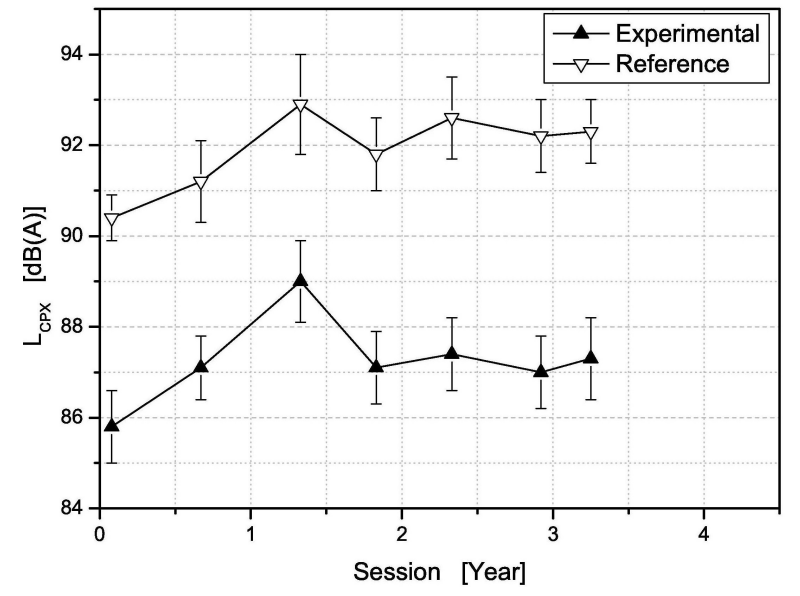

(a)

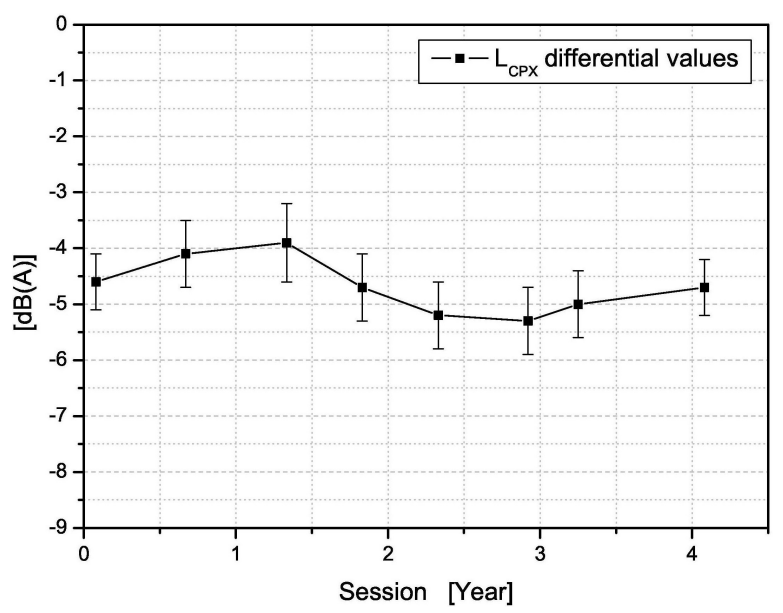

(b)

Figure 9. Absolute (a) and differential (b) values of $L_{C P X}$ along measurement sessions (e.g., time, in years) for the ISO10844 optimized texture surface laid in Site 3.

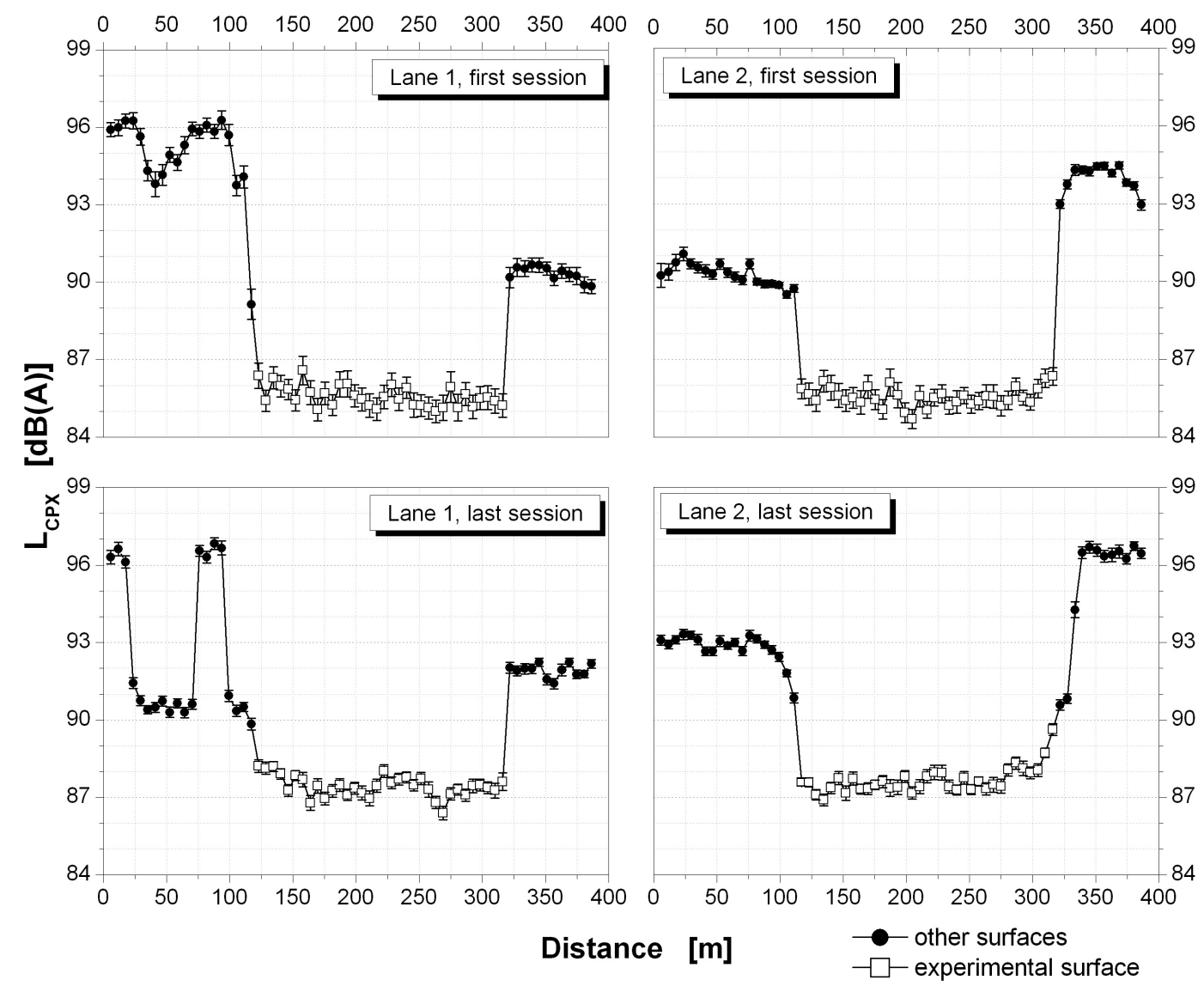

Figure 10. CPX data spatial distribution obtained during first session (upper figures) of measurements and during last one (lower figures) at Site 3. Lane 1 results are represented on the left plots while lane 2 ones are on the right plots. Experimental stretches data are marked with white squares.

Even if CPX and SPB do not show a clear relationship, the SPB results shown in Figure 11 confirm this good performance. The difference of about 2-3 $\mathrm{dB}(\mathrm{A})$ between the two SPB microphones is probably due to the absorbing lawn influence. 


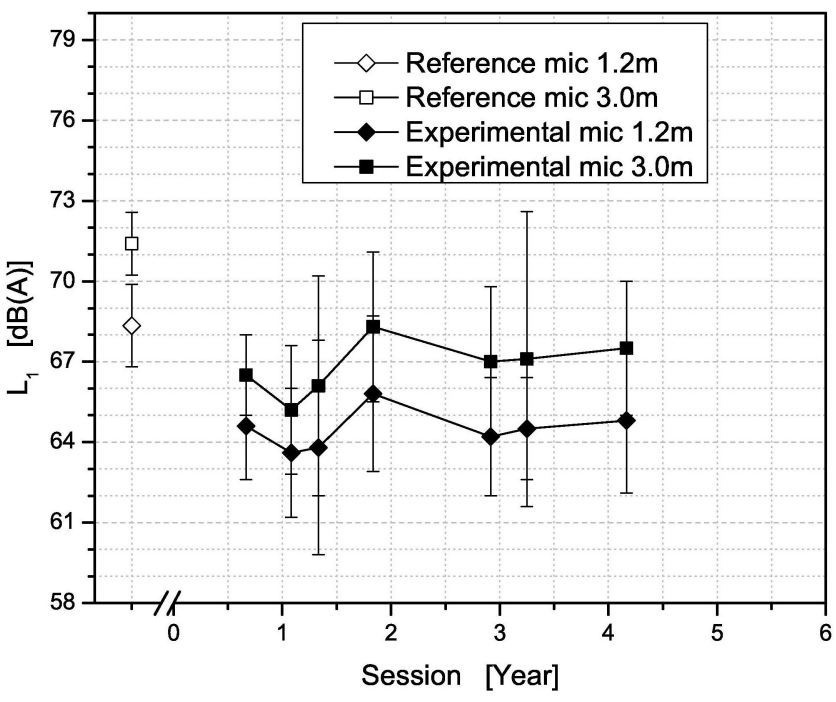

Figure 11. Time evolution of SPB values in Site 3. In white ante-operam values.

\subsection{Site 4-The Dense Grade 0/6 with Expanded Clay}

This straight site presents a high traffic density with high percentage of heavy vehicles and an average speed of about $80 \mathrm{~km} / \mathrm{h}$. No slope is present over this road, with open plane fields on one side and a slight ascending slope of the ground on the other.

The CPX results are shown in Figure 12, the SPB results are shown in Figure 13. In this case, results are highly correlated among the two methods and they show clearly a decaying of the acoustical characteristics of the road surface. According CPX method results decaying might be flattening at around $3 \mathrm{~dB}(\mathrm{~A})$ of tire/road noise lowering. Despite the high data correlation shown with the CPX data with, SPB results are not able to assess this lowering by means of the comparison with the ante-operam value.

The CPX data spatial distributions shown in Figure 14 point out the homogeneity stability since the first measurement session and till last one, showing the clearly increasing levels, similar for both lanes.

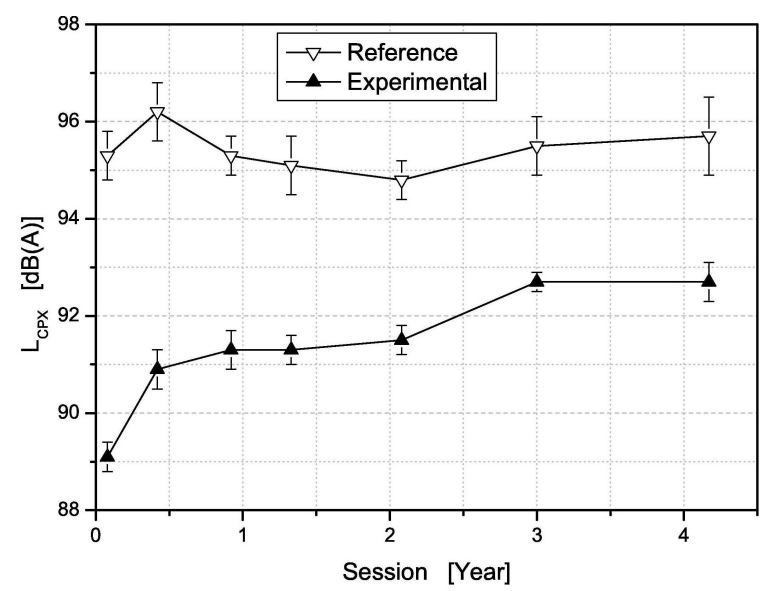

(a)

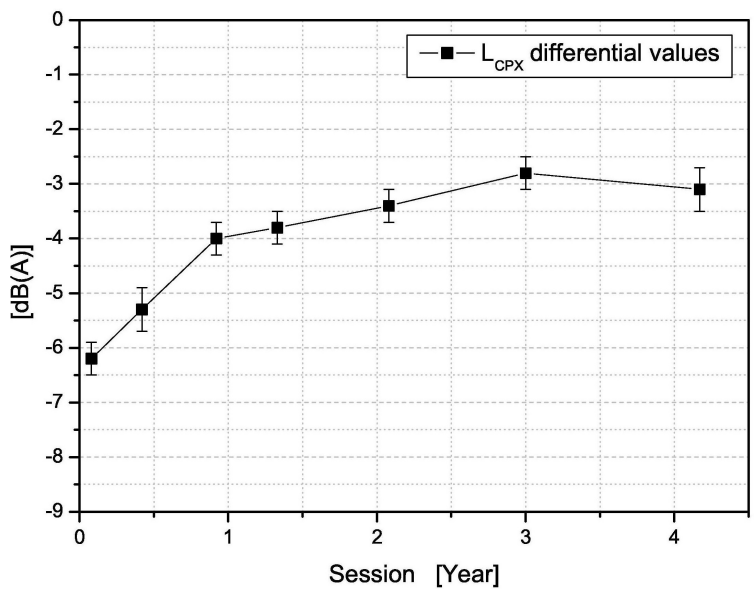

(b)

Figure 12. Absolute (a) and differential (b) values of $L_{C P X}$ along measurement sessions (e.g., time, in years) for the Dense grade 0/6 with expanded clay laid in Site 4. 


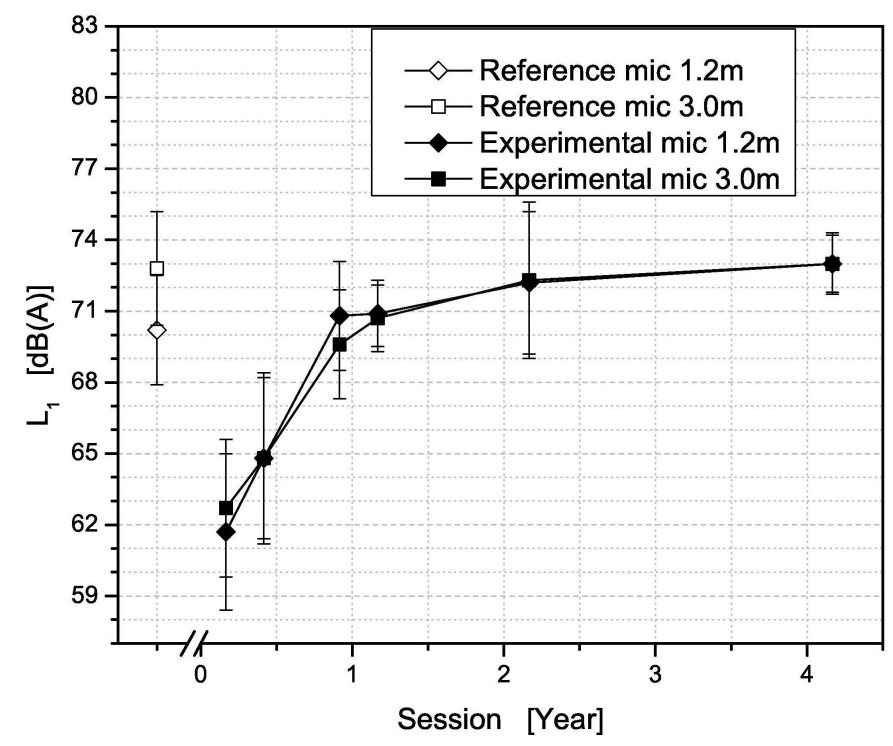

Figure 13. Time evolution of SPB values in Site 4. In white ante-operam values.
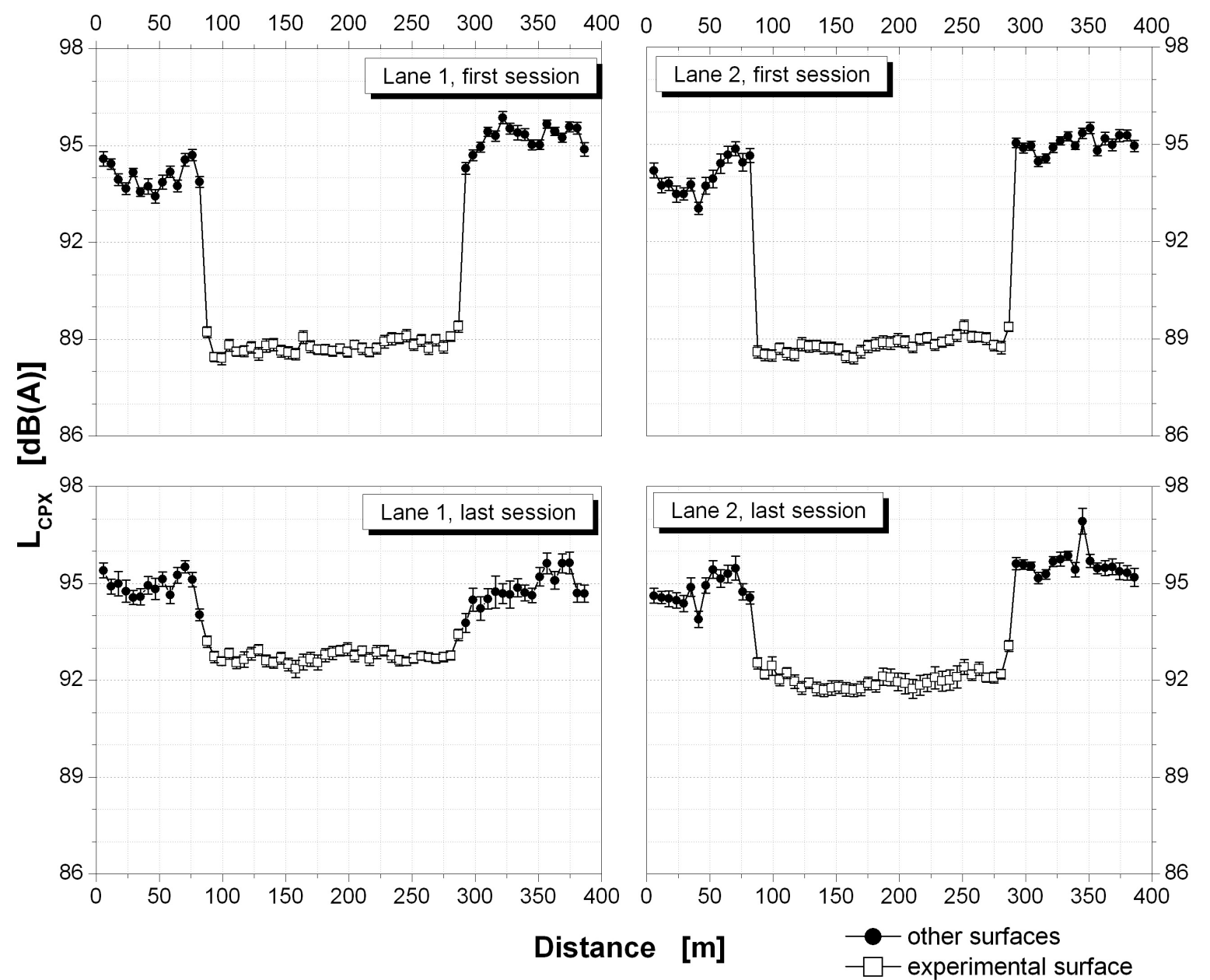

Figure 14. CPX data spatial distribution obtained during first session (upper figures) of measurements and during last one (lower figures) at Site 4. Lane 1 results are represented on the left plots while lane 2 ones are on the right plots. Experimental stretches data are marked with white squares. 


\subsection{Site 5-The Micro-Draining Open Grade 0/6}

This road has very low traffic density, but with a significant percentage of heavy vehicles during daytime. Bends are present with a slow slope and lanes are less than $3.5 \mathrm{~m}$ width, and the average speed is lower than $50 \mathrm{~km} / \mathrm{h}$. The ground on one side of the road shows a depression, while SPB instrumentation is placed on a wide parking lot on the other side.

The experimental road surface is porous and it shows an absorbing peak around $800 \mathrm{~Hz}$, as shown in Figure 15.

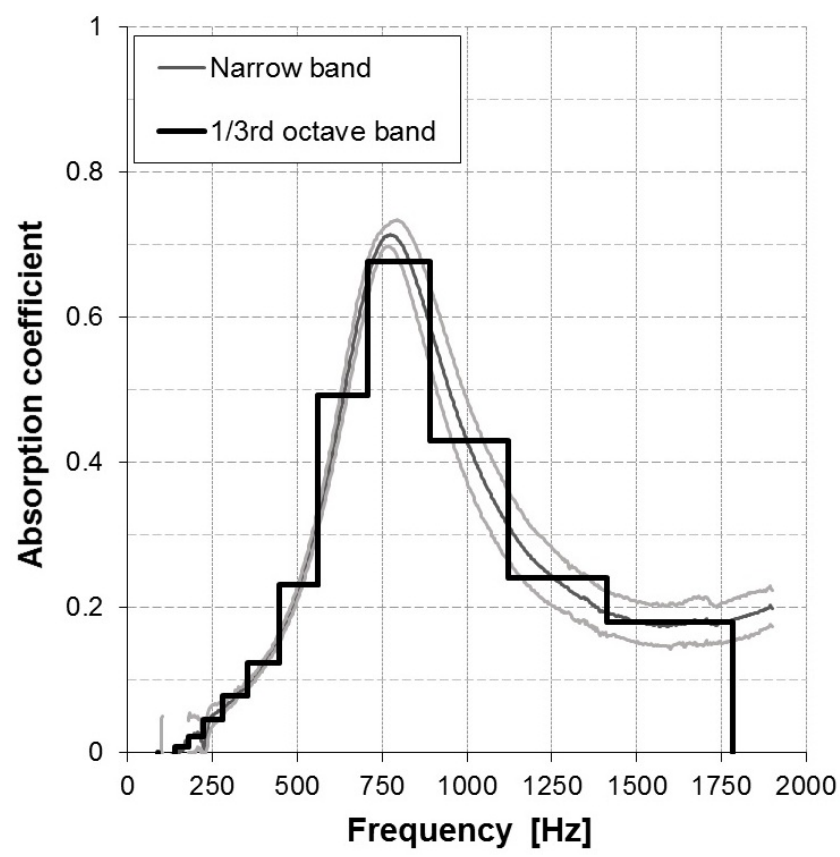

Figure 15. Site 5: Absorbing coefficient vs. frequency measured using impedance tube on some samples extracted from the surface. In gray the dispersion of data from narrow band analysis and in black the resulting 1/3rd octave band result. The main absorption peak can be found at about $800 \mathrm{~Hz}$.

The traffic density and average speed are too low to maintain the porosity, and therefore the acoustical characteristics are steeply decaying, even though from an optimal initial $8 \mathrm{~dB}(\mathrm{~A})$ lowering of tire/road noise emission, as well shown by the CPX results in Figure 16.

In Figure 17 the data spatial distribution of both lanes is shown, pointing out high unhomogeneity since the first measurement session, probably due to troubles in the laying. In particular, lane 2 shows a down-step shape still detectable in the last session. On lane 1 higher homogeneity can be found except at borders. It is worth noting, however, that the road surface shows a stretch per lane (lane 1 between 180 and $320 \mathrm{~m}$, lane 2 between 280 and $350 \mathrm{~m}$ ) with very low levels even after four years.

On the SPB results (see Figure 18), it has to be pointed out that sample data were subjected to strong statistical fluctuations, due to the low traffic density, which imply both a strong variability due to driving behavior and a gathering at the same speed. As well described above, these sample fluctuations highly influence the fit algorithm and the SPB results do not show the relationship with CPX ones. 


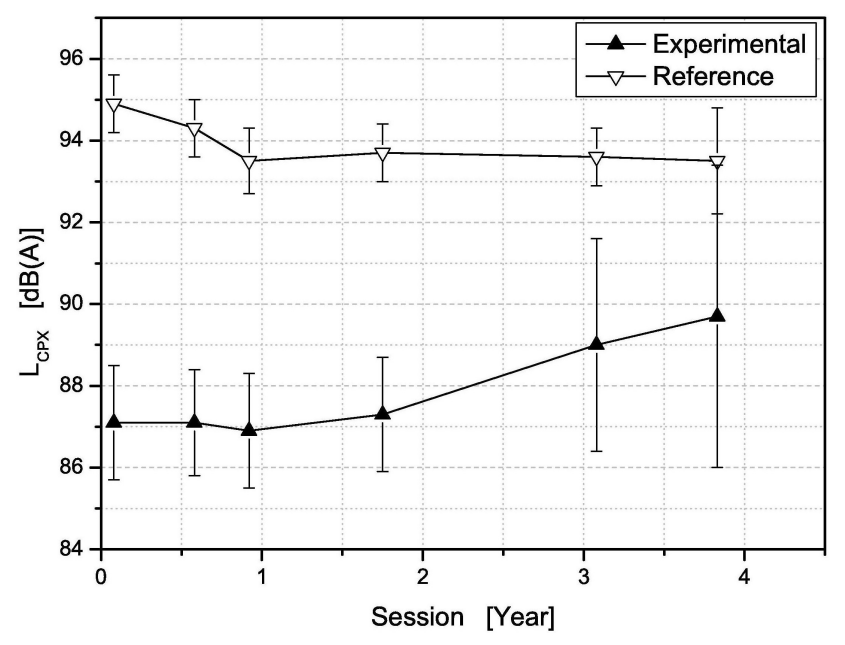

(a)

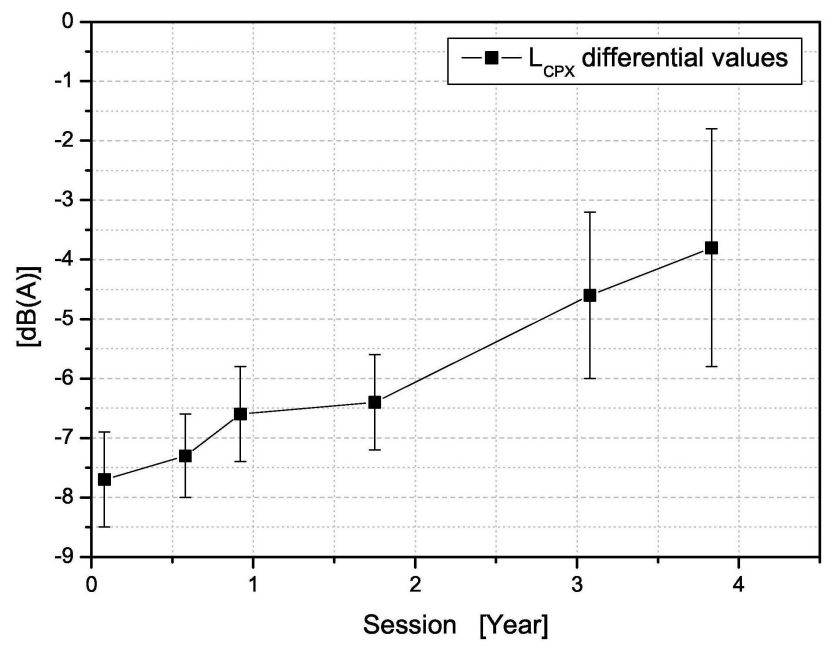

(b)

Figure 16. Absolute (a) and differential (b) values of $L_{C P X}$ along measurement sessions (e.g., time, in years) for Micro-draining open grade surface laid in Site 5.

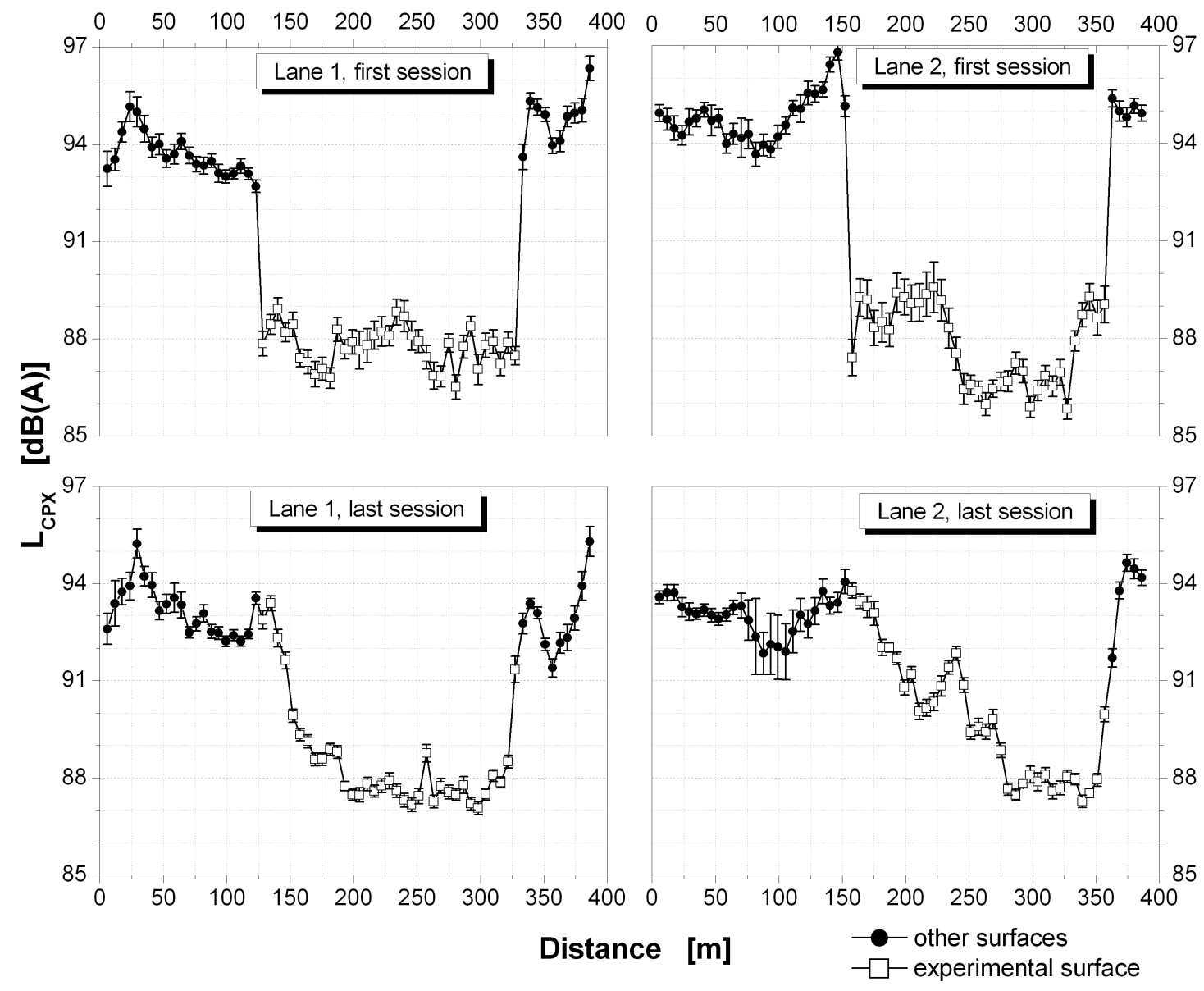

Figure 17. CPX data spatial distribution obtained during first session (upper figures) of measurements and during last one (lower figures) at Site 5. Lane 1 results are represented on the left plots while lane 2 ones are on the right plots. Experimental stretches data are marked with white squares. 


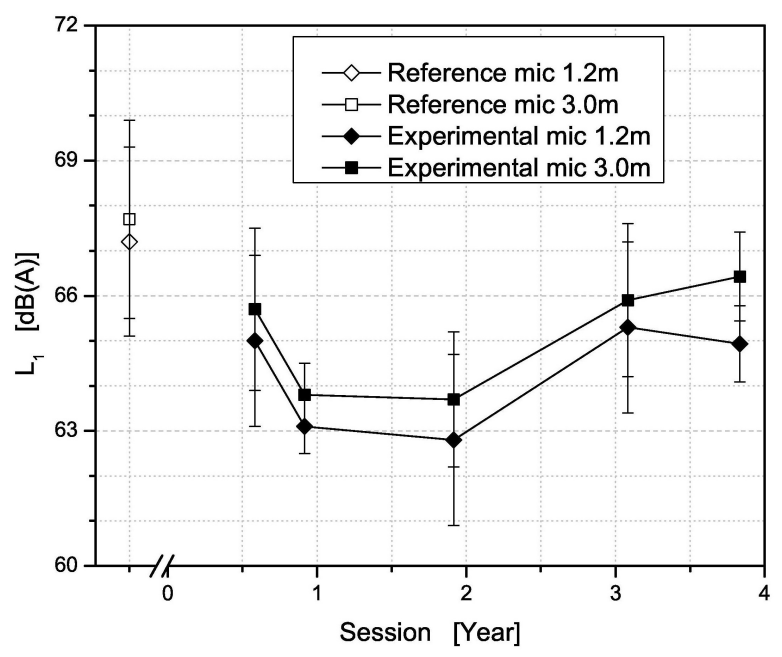

Figure 18. Time evolution of SPB values in Site 5. In white ante-operam values.

\subsection{Site 6-The Asphalt Rubber (Wet Process) Gap Grade 0/8}

Medium traffic density, with a significant percentage of heavy vehicles and an average speed of about $70 \mathrm{~km} / \mathrm{h}$ characterize this site, whose wide lanes allow good visibility. The ground around the road is a lawn almost flat on the side where the SPB instrumentation is placed, whilst the other side is a steep descending slope.

The CPX results are shown in Figure 19. In this case, the reference surface used to apply the differential value is coeval with the experimental one (results obtained using a per-existing surface as reference are shown in [9]). Looking at the absolute values, the reference surface probably cannot yet be considered acoustically settled, while the experimental road surface shows a strong time-stability. Therefore, the $L_{C P X}$ differential values are increasing and the lowering of tire/road noise emission is clearly improving.

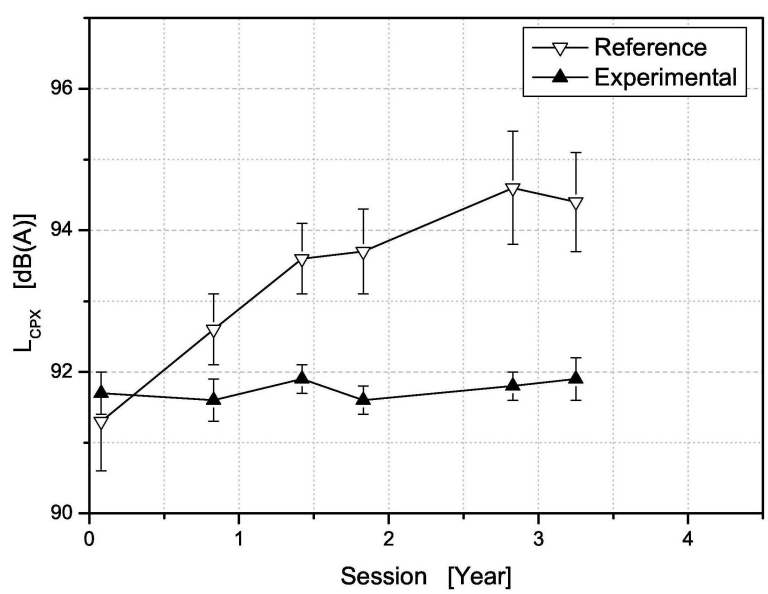

(a)

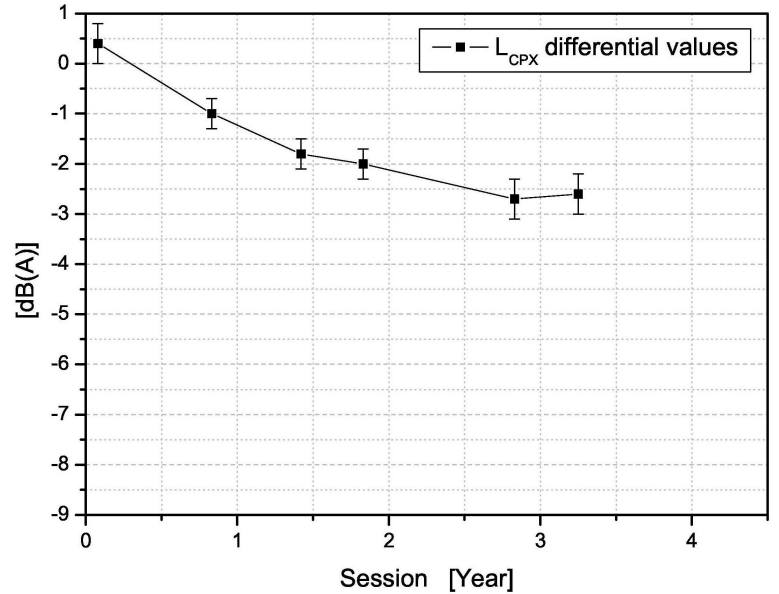

(b)

Figure 19. Absolute (a) and differential (b) values of $L_{C P X}$ along measurement sessions (e.g., time, in years) for Asphalt rubber (wet process) surface laid in Site 6.

The CPX spatial distribution, shown in Figure 20, points out its homogeneity and its stability in time: since the first measurement session and till the last one, lanes show equivalent levels. 
The SPB results (see Figure 21) confirm the good stability of the experimental road surface. The difference of about 2-3 $\mathrm{dB}(\mathrm{A})$ between the two SPB microphones is probably due to the absorbing lawn influence, as in case of site 3. It has to be underlined that for the SPB the reference surface was the per-existing one.

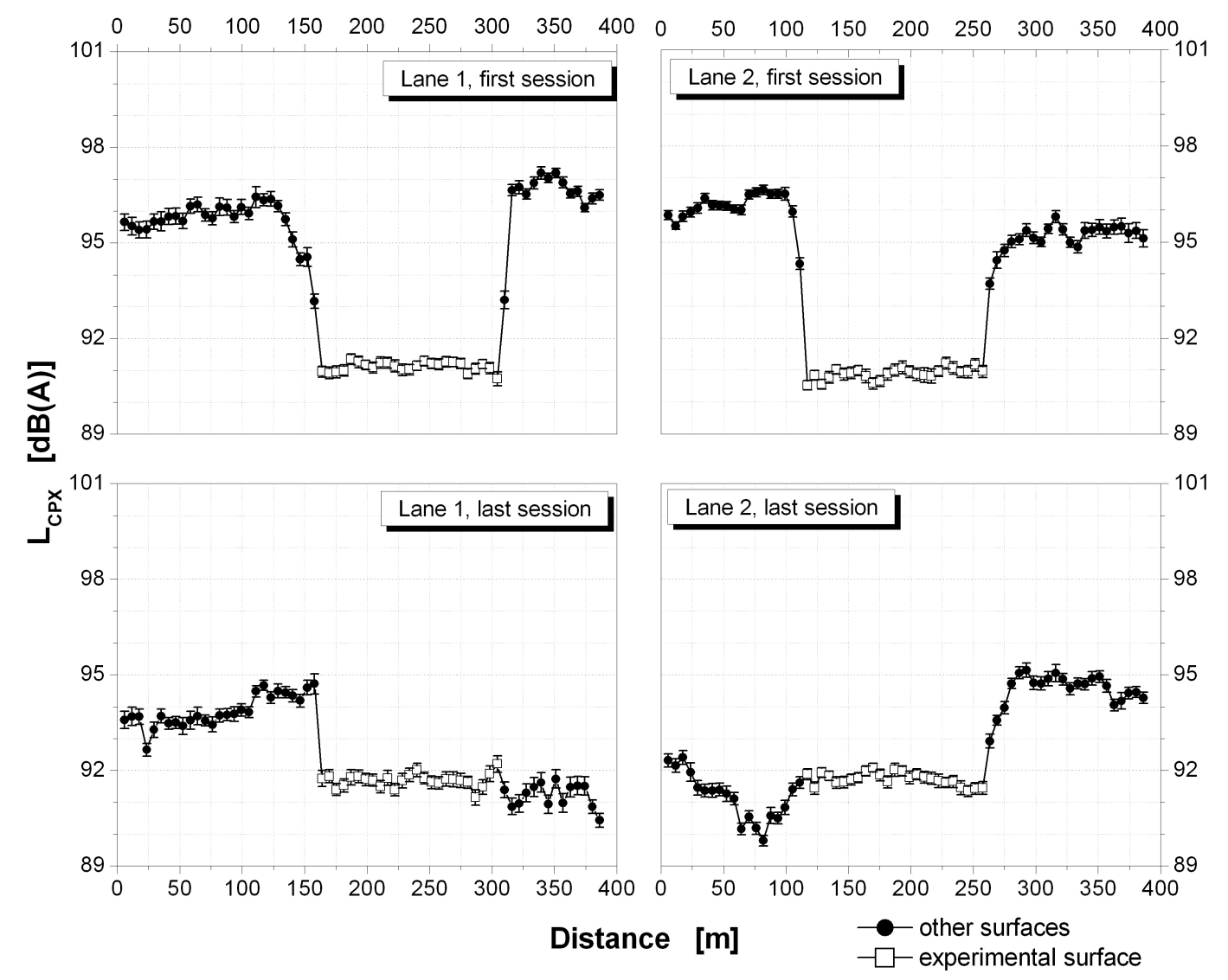

Figure 20. CPX data spatial distribution obtained during first session (upper figures) of measurements and during last one (lower figures) at Site 6. Lane 1 results are represented on the left plots while lane 2 ones are on the right plots. Experimental stretches data are marked with white squares.

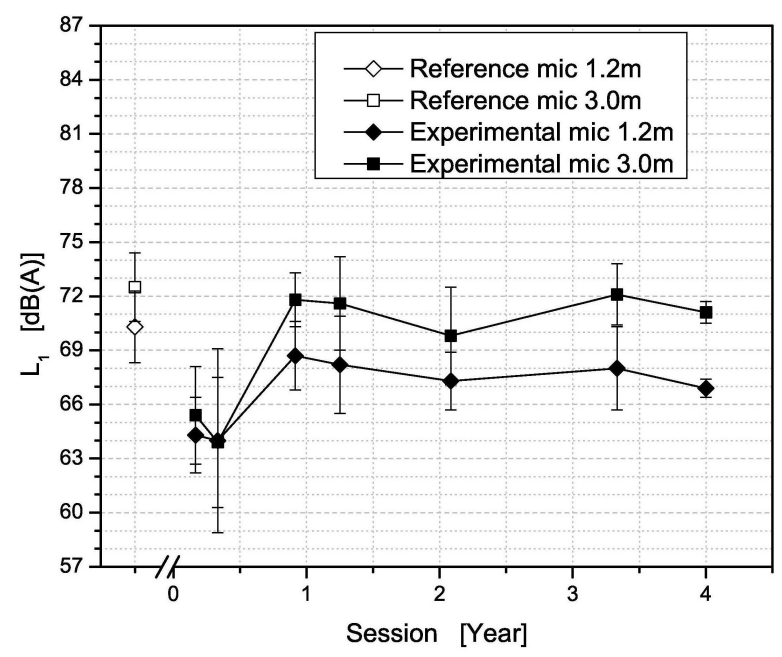

Figure 21. Time evolution of SPB values in Site 6. In white ante-operam values. 


\section{Discussion}

The most challenging task for the LEOPOLDO project was studying and developing methods and protocols useful to assess the effectiveness of the mitigation action planned by means of road surfaces. Indeed, when somewhere there is an excess of noise limit a mitigation action is needed.

The mitigation action should be a solution stable in time as much as possible, to avoid recurring of the noise limit excess and incurring further costs. The main critical issue of the verifications requested by laws are that they are carried out just in few points, or even in only one, and that the time monitoring is not provided for. It means that regulation criteria are not able to leave spatial homogeneity and time stability out of consideration. On the contrary, it is well-known that every road surface is weathered and exposed to traffic: resulting wear and tear corresponds to a worsening of acoustical performances. Moreover, many road surface technologies prescribe specific production methods, that if unattended leads to inhomogeneity in case of very long laying. Thus, choosing the right road surface to be used in the mitigation action planning phase does not guarantee the expected outcome, neither in terms of spatial regularity nor in terms of time durability.

A second critical issue is the ability of identifying which action has failed when the limit excess is assessed after the realization of a mitigation project planned with several actions combined together. In fact, the verifications requested by laws are not able to provide the answer. Similarly, when the excess of noise limit recurs after some time, it is necessary to understand if the cause is the decaying of the road surface acoustical characteristics or if the site conditions are changed (for example an increasing of traffic density or of heavy vehicles, etc.).

Therefore, it is necessary to have a measurement protocol which is able to evaluate the road surface effectiveness, its time evolution and without being influenced by traffic conditions, noise barriers etc. The experience gained within LEOPOLDO project leads to identify the CPX method, applied with the differential criterion, as the most suitable protocol to test a road surface, in space and in time. Choosing the most appropriate reference surface to apply the differential criterion is crucial, especially to the time stability investigation. For example, in the LEOPOLDO project even if the reference surfaces used are all of the same type commonly used in Tuscany, they provide different $L_{C P X}$ absolute values varying in about $4 \mathrm{~dB}(\mathrm{~A})$. This does not allow the comparison between different road surface types by means of the $L_{C P X}$ differential values, and only the comparison between mitigation actions is allowed.

On the other side, CPX analysis allows to assess when SPB data are too influenced by surrounding conditions, e.g., see in site 1 . Moreover, $L_{C P X}$ values as a function of the distance allows to evaluate the surface spatial regularity, with a measurement time significantly shorter than which requested by roadside methods.

The SPB method is not able to evaluate the road surface homogeneity. In addition, results are useful just to describe the stretch in front of the measurement position and they cannot be considered representative of the whole installation when highly spatial inhomogeneous, as in case of Site 1.

Being the laying homogeneous, the SPB method could be useful to compare different road surface types, but it suffers the sample variability, which corresponds to high associated uncertainties. Moreover, without a reference data set (provided from a reference surface or from the CPX method, as in case of the LEOPOLDO project), it suffers too much the surrounding conditions. The too high uncertainties 
and the possibility to chance upon an outlier due to surrounding conditions smooth over the SPB values, masking the aimed information. In particular this may be crucial when influencing results of ante-operam measurement (see site 4). Anyway, the SPB method has the advantage to use the actual car fleet as source, whilst the CPX method uses just one or few particular tires, and the gain in low tire/road noise assessed by means of the CPX method not necessarily is well representative of the roadside benefit due to the surface laid on an ordinary road.

\section{Conclusions}

In Tuscany, the LEOPOLDO project was planned in order to develop innovative noise mitigation techniques to be used in action plans for road infrastructures, based on new type of pavement layers: the project implementation is part of the required environmental policies to mitigate road noise pollution implemented by the Tuscany Region and other European Community member states, in accordance with the Directive of the European Parliament and Council 2002/49/EC [6].

The first task of the LEOPOLDO project was to study experimental low noise road surfaces, taking into account also the environmental compatibility, in order to define criteria on which choosing the most suitable surface to use when a noise mitigation action is needed. Thus, six experimental road surfaces have been proposed, laid in different contexts and monitored over four years. The performance of low-noise road surfaces has been monitored using three measurement methods, aiming to evaluate their suitability for a correct assessment of the effectiveness of a pavement laid as mitigation planning. Evaluation of roadside noise through the SPB method has shown unreliable results due to the influence of surrounding conditions, even using a modified procedure. Results have pointed out that the best evaluation of surfaces performance in the lowering of road/tire noise is achieved through CPX method and a modified measurement protocol that evaluate differential values in order to assure the comparability of performances along time.

The experience of the LEOPOLDO project improved the knowledge in the design and the characterization of low-noise road surfaces. And a further project—named LEOPOLDO II-is ongoing in order to develop further skills.

\section{Acknowledgments}

Results come from measurement sessions performed within the Leopoldo Project funded by Transportation Ministry and Tuscany Region. This paper could not be written to its fullest without the support of different funds and people that supported the analysis within these 8 years. Special thanks to Luca Alfinito, Luca Nencini and Riccardo Zei for their precious work.

After the official end of the project further analysis become possible with the financial support of Ecopneus s.c.p.a., which funded a research project carried out by CNR-IPCF and its spin-off iPOOL s.r.l. 


\section{Author Contributions}

Experimental measurements, analysis and interpretation of the results as well as conclusions have been conducted by all the co-authors. The manuscript has been written by Luca Teti, artworks have been prepared by Mauro Cerchiai, the whole paper has been revised by Gaetano Licitra with approval by the others co-authors.

\section{Conflicts of Interest}

The authors declare no conflict of interest.

\section{References}

1. Sandberg, U.; Ejsmont, J.A. Tyre/Road Noise Reference Book; INFORMEX: Kisa, Sweden, 2002.

2. Sandberg, U.; Kalman, B.; Nilsson, R. SILVIA Project Report-Design Guidelines for Construction and Maintenance of Poroelastic Road Surfaces; Project Deliverable SILVIAVTI-005-02-WP4-141005, SILVIA-CONTRACT N-GRD2-2000-31801-SI2.335701; Swedish National Road and Transport Research Institute (VTI): Linköping, Sweden, 2005.

3. Harmonised, Accurate and Reliable Prediction Methods for the EU Directive on the Assessment and Management Of Environmental Noise; IST-2000-28419; European Commission: Utreche, The Netherlands, 2000.

4. Improved Methods for the Assessment of the Generic Impact of Noise in the Environment; SSPI-CT-2003-503549-IMAGINE; Netherlands Organisation for Applied Scientific Research (TNO): Delft, The Netherlands, 31 May 2006.

5. Predisposizione Delle Linee Guida Per La Progettazione Ed Il Controllo Delle Pavimentazioni Stradali Per La Viabilità Ordinaria; University of Pisa: Pisa, Italy, 2006. (In Italian)

6. The Environmental Noise Directive; 2002/49/EC; European Commission: Brussels, Belgium, 2002.

7. Licitra, G.; Cerchiai, M.; Teti, L.; Alfinito, L. Road pavement description by psycho-acoustical parameters from CPX data. In Proceedings of the 38th International Congress and Exposition on Noise Control Engineering (INTER-NOISE 2009), Ottawa, Canada, 23-26 August 2009.

8. Licitra, G.; Cerchiai, M.; Alfinito, L. Acoustical performances of new generation road pavements developed in LEOPOLDO Project. In Proceedings of the 8th European Conference on Noise Control (Euronoise 2009), Edinburgh, UK, 26-28 October 2009.

9. Licitra, G.; Cerchiai, M.; Teti, L.; Ascari, E.; Fredianelli, L. Durability and variability of the acoustical performance of rubberized road surfaces. Appl. Acoust. 2015, in press.

10. Ascari, E.; Licitra, G.; Cerchiai, M.; Teti, L. Low frequency noise impact from road traffic according to different noise prediction methods. J. Sci. Total Environ. 2015, 505, 658-669.

11. Losa, M.; Leandri, P.; Licitra, G. Mixture design optimization of low-noise pavements. J. Transp. Res. Board 2013, 23-72, 25-33.

12. Losa, M.; Leandri, P.; Cerchiai, M. Improvement of pavement sustainability by the use of crumb rubber modified asphalt concrete for wearing courses. Int. J. Pavement Res. Technol. 2012, 5, 395-404. 
13. DELIBERAZIONE di Giunta Regionale 11 marzo 2013, n. 157, BURT part 2-n.12 20-3-2013; Regione Toscana: Florence, Italy, 2013. (In Italian)

14. Delibera Giunta Regionale n.490 16-06-2014. Comitato regionale di coordinamento ex art. 15 bis, L.R. 89/98: linee guida regionali in materia di gestione degli esposti, di verifica di efficacia delle pavimentazioni stradali fonoassorbenti elo a bassa emissività negli interventi di risanamento acustico e di gestione dei procedimenti di Valutazione di Impatto Acustico; ARPAT (Agenzia regionale per la protezione ambientale della Toscana): Florence, Italy, 2014. (In Italian)

15. ISO 11819-1 Acoustics-Measurement of the Influence of Road Surfaces on Traffic Noise-Part 1: Statistical Pass-By Method; ISO: Geneva, Switzerland, 1997.

16. ISO/DIS-11819-2 Method for Measuring the Influence of Road Surfaces on Traffic Noise-Part 2: Close-Proximity (CPX) Method; ISO: Geneva, Switzerland, 2011.

17. ISO 10534-1: 1996 Acoustics_Determination of Sound Absorption Coefficient and Impedance in Impedance Tubes_Part 1: Method Using Standing Wave Ratio; ISO: Geneva, Switzerland, 1996.

18. ISO 10534-1: 1998 Acoustics_Determination of Sound Absorption Coefficient and Impedance in Impedance Tubes_Part 2: Transfer-Function Method; ISO: Geneva, Switzerland, 1998.

19. Jonasson, H. Test Method for the Whole Vehicle; Technical Report HAR11TR-020301-SP10; 2004

20. ISO 1996-2:2010: Acoustics-Description, Measurement and Assessment of Environmental Noise_Part 2: Determination of Environmental Noise Levels; ISO: Geneva, Switzerland, 2010.

21. Licitra, G.; Teti, L.; Cerchiai, M. A modified Close Proximity method to evaluate the time trends of road pavements acoustical performances. Appl. Acoust. 2014, 76, 169-179.

22. Bollard, K. Report on the Status of Rubberized Asphalt Traffic Noise Reduction in Sacramento County; County of Sacramento Public Works Agency-Transportation Division: Sacramento, CA, USA, 1999.

23. Bucka, M. Asphalt Rubber Overlay Noise Study Update; Technical Report AAAI Report 1272; County of Sacramento Public Works Agency: Sacramento, CA, USA, December 2002.

24. Treleaven, L.; Pulles, B.; Bilawchuk, S.; Donovan, H. Asphalt rubber-The quiet pavement? In Proceedings of the Annual Conference and Exhibition of the Transportation Association of Canada, Charlottetown, Canada, 17-20 September 2006.

(c) 2015 by the authors; licensee MDPI, Basel, Switzerland. This article is an open access article distributed under the terms and conditions of the Creative Commons Attribution license (http://creativecommons.org/licenses/by/4.0/). 